\title{
EMBEDDABILITY OF DISCRETE TIME SIMPLE BRANCHING PROCESSES INTO CONTINUOUS TIME BRANCHING PROCESSES( $\left.{ }^{1}\right)$
}

\author{
BY \\ SAMUEL KARLIN AND JAMES MCGREGOR
}

0. Introduction. A simple discrete time Markoff branching process $\left\{X_{n}, n \geqq 0\right\}$ with state space the nonnegative integers is induced by a probability generating function (abbreviated hereafter as p.g.f.) $f(x)=\sum_{k=0}^{\infty} a_{k} x^{k}$ where $a_{k} \geqq 0, k=0,1,2$, $\ldots, f(1)=1$. The transition probability matrix

$$
P_{i j}=\operatorname{Pr}\left\{X_{n+1}=j \mid X_{n}=i\right\}
$$

is determined from the generating function by the relations

$$
\sum_{j=0}^{\infty} P_{i j} x^{j}=[f(x)]^{i}, \quad i=0,1,2, \ldots
$$

The p.g.f. $f(x)$ is interpreted as the number of progeny produced by each parent per generation. All offspring act independently with the same fixed lifetime and the same distribution of progeny.

It is a familiar fact that the $n$ step transition probability matrix

$$
P_{i j}^{(n)}=\operatorname{Pr}\left\{X_{n}=j \mid X_{0}=i\right\}
$$

possesses the generating function

$$
\sum_{j=0}^{\infty} P_{i j}^{(n)} x^{j}=\left[f_{n}(x)\right]^{i}
$$

where $f_{n}(x)=f_{n-1}(f(x))$ is the $n$th functional iterate of $f(x)$. The branching process is called critical, supercritical or subcritical according as $f^{\prime}(1)=m=1, m>1$ or $m<1$. For our purposes a one-dimensional continuous time Markov branching process $\left\{Z_{t}, t \geqq 0\right\}$ is a temporally homogeneous continuous time Markov chain with the nonnegative integers as state space, characterized by the following properties: (i) the p.g.f.'s $f_{t}(x)=\sum_{k=0}^{\infty} x^{k} \operatorname{Pr}\left\{Z_{t}=k \mid Z_{0}=1\right\}$ continuous in $t \geqq 0$ and $|x| \leqq 1$ are related functionally in the manner

$$
f_{t+s}(x)=f_{t}\left(f_{s}(x)\right), \quad t, s \geqq 0,|x| \leqq 1,
$$

Received by the editors February 13, 1967.

(1) Research supported in part under Contracts NO 014-67-A-0112-0015 and NIH USPHS 10452 at Stanford University. 
(ii) individual particles generate independent lines of descent, i.e.,

and

$$
\sum_{k=0}^{\infty} x^{k} \operatorname{Pr}\left\{Z_{t}=k \mid Z_{0}=i\right\}=\left[f_{t}(x)\right]^{i}
$$

(iii) $f_{t}(1)=1$ for all $t \geqq 0$.

Condition (iii) means that we only deal with conservative (nonexplosive) continuous time branching processes.

Given the above formulation the semigroup $f_{t}(x)$ is uniquely determined by the infinitesimal generating function $u(x)$ connected to $f_{t}(x)$ through the backward equation

$$
\left(\partial f_{t} / \partial t\right)(x)=u\left(f_{t}(x)\right), \quad f_{0}(x)=x .
$$

Moreover, the associated infinitesimal generating function has the form $u(x)=$ $\lambda[h(x)-x]$ where $h(x)=\sum_{i=0}^{\infty} p_{i} x^{i}, p_{i} \geqq 0, i=0,1, \ldots, \sum_{i=0}^{\infty} p_{i}=1$ and $\lambda>0$. As usual, when dealing with continuous time branching processes, we assume $h^{\prime}(1)<\infty$. This hypothesis will be mostly redundant, emerging as a consequence of the considerations at hand.

The value of $h^{\prime}(1)$ can be interpreted as the expected number of progeny produced by a parent at each split. The lifetime of each individual is exponentially distributed with parameter $\lambda^{-1}$.

Inspection of the definitions reveals that $X_{n}=Z_{n \delta}, n=0,1, \ldots$, for each fixed $\delta>0$, generates a discrete time Markoff branching process provided $Z_{t}, t \geqq 0$, describes a continuous time Markoff branching process as defined above. We will investigate the converse problem concerned with delimiting criteria to ascertain when a p.g.f. $f(x)$ inducing a discrete time branching process be embeddable in a continuous time Markoff branching process. The concept of embeddability is made precise by the following definition.

Definition 1. A p.g.f. $f(x)$ is said to be embeddable if there exists a one parameter semigroup of p.g.f.'s $f_{t}(x)$, continuous in $0 \leqq t<\infty,|x| \leqq 1$, such that

$$
f_{t+s}(x)=f_{s}\left(f_{t}(x)\right), \quad t, s \geqq 0,|x| \leqq 1
$$

and

$$
f_{1}(x)=f(x)
$$

The class of embeddable p.g.f.'s is denoted by $\mathscr{I}$.

Applications involving discrete time branching processes frequently entail the postulate that the progeny p.g.f. $f(x)$ is of a classical type (Poisson, binomial negative binomial and similarly). We will find that none of these examples (the single exception being a linear fractional function) is embeddable. On the other hand, if the infinitesimal generating function $u(x)=\lambda(h(x)-x)$ of a continuous time Markov branching process is stipulated to be of a classical kind then the corresponding set of p.g.f.'s $\left\{f_{t}(x)\right\}$ determined from $u(x)$ are generally not of 
recognizable form. This striking disparity poses fundamental questions as to whether continuous or discrete time units are most appropriate in the formulation of branching process models. These considerations affect the nature of the assumptions made on the progeny distributions especially pertaining to applications in genetics and in the study of electron, photon cascade showers.

The results derived in this paper are mostly of a negative kind. We present several theorems indicating that large classes of p.g.f.'s of the kind commonly occurring in practice are nonembeddable. In $\S 1$ of this paper we set up the basic background material and state several of the principal theorems of the paper. $\$ 2$ develops a variety of technical facts concerning infinitesimal generating functions and their relations to some natural functional equations intrinsic to the theory of functional iterations of a given function. Several of the results of this section provide important analytic properties of relevance in the study of the structure of supercritical branching processes (e.g., see [3] and [7]).

A characterization of the linear fractional and some cognate p.g.f.'s from the class $\mathscr{I}$ is set forth in $\S 3$.

$\$ 4$ is devoted primarily to the proof of Theorem 6 which shows that certain classes of p.g.f.'s having branch point singularities are nonembeddable. This result nicely supplements the principal theorem of Karlin and McGregor [8] which asserts that iff is single valued and possesses at most a countable number of singularities in the extended complex plane then $f$ is embeddable if and only if $f$ is a linear fractional function. (See also Theorem A of $\S 1$.) In $\$ 4$ we also establish limitations on the growth behavior of $f$ in $\mathscr{I}$ when $f(x)$ is regular at 1 .

Some special criteria for nonembeddability are discussed in $\S 5$. These criteria are frequently easily verifiable for large classes of p.g.f.'s. In $\$ 6$ the connections of embeddability of $f$ and univalent mapping properties for $f$ are elaborated.

We close this introduction with a brief survey of the related literature of the subject. Harris [2] proved that if a p.g.f. $f$ is entire, and $f(0)=0$, then $f$ is nonembeddable. This fact is, of course, a very special case of Theorem A (see $\$ 1$ ). In his book Harris [3, pp. 102-103] raises the problem of determining conditions for embeddability. This paper to a great extent settles the problem for those functions $f$ of practical interest, mostly in a negative sense.

The question of embedding an analytic function $g(x)$ with a fixed point $x^{*}$ in its domain of regularity into a one parameter group $g_{t}(x), t \geqq 0$, each analytic near $x^{*}$ (discarding the requirement that $g_{t}(x)$ be a p.g.f. for each $t>0$ ) has a long history tracing back to Fatou, e.g., see Koenigs [9]. This problem is of interest in the theory of conformal mapping, iterative processes of analysis and elsewhere. Recently, numerous authors have studied various facets of the general problem of whom we mention only Baker [1], Jabotinsky [4], Szekeres [11], and Karlin and McGregor [8].

1. Preliminaries and statement of results. Let $f(x)$ be a p.g.f. Henceforth, we 
exclude from all future considerations the case where $f(x)=a_{0}+a_{1} x$ is a linear function. The relevant results for this case are trivial.

It is convenient to review some of the elementary facts concerning the supercritical $(m>1)$ and subcritical $(m<1)$ cases. When $m>1$ there exists two fixed points $\pi$ and 1 of the transformation $y=f(x)$ with $0 \leqq \pi<1$. We fix the following notation

$$
\begin{gathered}
f(\pi)=\pi, \quad 0 \leqq \pi<1 ; \quad f(1)=1, \quad f^{\prime}(\pi)=c, \quad f^{\prime}(1)=m, \\
\alpha=\log (1 / c), \quad a=\log m, \quad \gamma=\alpha / a .
\end{gathered}
$$

Clearly $0 \leqq c<1<m$ and by definition $f(\pi)=\pi$ and $f(1)=1$. Moreover, $c>0$ provided $\pi>0$. However, if $\pi=0$ we assume $f^{\prime}(0)=c>0$. The case $f(0)=f^{\prime}(0)=0$ is nonembeddable by trivial arguments.

When $m \leqq 1$ (subcritical or critical cases), then 1 is the only root of $f(x)=x$ on the segment $0 \leqq x \leqq 1$. In the subcritical case it may happen (e.g., if $f(x)$ is meromorphic) that a second fixed point $\tilde{\pi}>1$ exists $(f(\tilde{\pi})=\tilde{\pi})$ such that $f(x)$ is regular in the disc $|x|<\tilde{\pi}+\varepsilon$, for some $\varepsilon>0$. The function

$$
g(x)=(1 / \tilde{\pi}) f(\tilde{\pi} x)
$$

is then a p.g.f. in the supercritical case with fixed points at $\pi=1 / \tilde{\pi}$ and 1 . Manifestly, $f(x)$ is embeddable iff $g(x)$ is. Henceforth the reduction (2) is performed whenever possible.

The transformation

$$
\hat{f}(x)=\psi^{-1}(f(\psi(x))), \quad \psi(x)=(1-\pi) x+\pi, \quad|x|<1,
$$

well defined in the supercritical case converts the fixed point $\pi$ into 0 , preserving the parameters

$$
c=\hat{f}^{\prime}(0)=f^{\prime}(\pi), \quad m=\hat{f}^{\prime}(1)=f^{\prime}(1) .
$$

Obviously, $f$ is embeddable iff $\hat{f}$ is embeddable. Henceforth, without restricting generality and unless stated explicitly otherwise, we may assume in the supercritical case that $f(0)=0$ (i.e., $\pi=0$ ).

We are now prepared to state some of the principal theorems of the paper. Because of the intrinsic differences in the nature of the assumptions we sometimes distinguish the three cases according as $f$ corresponds to the super, sub or critical cases. We cite first for reference and comparison the following theorem established in Karlin and McGregor [8].

THEOREM A. Let $f(x)$ be a p.g.f. regular at $x=1$ with $f^{\prime}(1)=m>1, f(0)=0$ and $f^{\prime}(0)=c>0$. Suppose the singular points of $f$ in the extended complex plane form a countable closed set $S$ and $f$ is single valued in the complement of $S$. Then $f$ is embeddable only if $f$ is a linear fractional function.

Theorem A tells us in particular that with one exception a meromorphic p.g.f. is nonembeddable. 
Definition 2. A function $f(x)$ with at most a countable closed set $S$ of singularities and single valued in the complement of $S$ is said to be of type $\mathscr{S}$.

In [8] a sharper result is proved to the effect that $f$ of type $\mathscr{S}$ is not only nonembeddable in the sense of Definition 1 but cannot even be embedded in a continuous one parameter semigroup of functions (not necessarily p.g.f.'s) $f_{t}(x), t \geqq 0$, which leaves invariant the segment $[0,1]$ except when $f$ is a linear fractional function.

Observe next that if we discard the single valuedness condition of Theorem A then numerous embeddable p.g.f.'s are available. The simplest class of explicit examples are the p.g.f.'s

$$
g(x ; k)=x(1-p)^{1 / k} /\left(1-p x^{k}\right)^{1 / k},
$$

where $k$ is a positive integer and $p$ is a parameter obeying the restriction $0<p<1$. We can express (5) in the form

$$
g(x ; k)=\varphi_{(k)}^{-1}\left(f\left(\varphi_{(k)}(x)\right)\right),
$$

where $\varphi_{(k)}(x)=x^{k}$ and $f(x)=(1-p) x /(1-p x)$. Since $f(x)=(1-p) x /(1-p x)$ is embeddable, viz.,

$$
f_{t}(x)=e^{-d t} x /\left(1-\left(1-e^{-d t}\right) x\right), \quad t \geqq 0,
$$

with $-d=\log (1-p)$ we find immediately the one parameter family of p.g.f.'s

$$
g_{t}(x ; k)=\varphi_{(k)}^{-1}\left(f_{t}\left(\varphi_{(k)}(x)\right)=\frac{e^{-d t / k} x}{\left(1-\left(1-e^{-d t}\right) x^{k}\right)^{1 / k}}\right.
$$

containing $g_{1}(x ; k)=g(x ; k)$.

The examples (5) possess a special extremal property clarified in Theorem 4 (§3). The following theorem singles out the functions of (5) and further complements the result of Theorem A for some cases of multivalued functions.

THEOREM 6. Let $f(x)$ be a p.g.f. regular at $x=1$ with $f(0)=0$ and $f^{\prime}(0)=c>0$. Let $x_{0}$ be the smallest positive singularity of $f$ exceeding 1 .

Suppose $x_{0}$ is an isolated singular point such that $f(x)$ admits a convergent expansion valid in a neighborhood about $x_{0}$ of the form

$$
f(x)=\frac{1}{\left(x-x_{0}\right)^{\delta}} \sum_{r=0}^{\infty} c_{r}\left(x-x_{0}\right)^{r}
$$

with $\delta>0$ and $c_{0} \neq 0$. Then $f$ is embeddable iff $f(x)=g(x ; k)$ for some positive integer $k$.

REMARK. The existence of $x_{0}$ is affirmed by appeal to Pringsheim's theorem (see $[12$, p. 214]).

A direct application of Theorem 6 confirms the fact that

$$
f(x)=(1-p)^{\alpha} /(1-p x)^{\alpha}, \quad \alpha>0,
$$

is nonembeddable except in the special case $\alpha=1$. 
In the process of the proof of Theorem 6 we also secure the following growth properties whenever $f$ is embeddable and regular at 1 .

Proposition 1. Let $f(x)$ be regular at 1 and embeddable. Suppose $1<x_{0}<\infty$ where $x_{0}$ is the smallest pasitive singularity of $f$. Then $f(x) \leqq C /\left(x_{0}-x\right)$ as $x \uparrow x_{0}$ where $C$ is an appropriate constant.

More generally, suppose $f(x)$ is embeddable with associated infinitesimal generating function $u(x)$. If $u(x)$ is a polynomial of degree $r(r \geqq 2)$, then $f$ is a multivalued function with first singularity at $x_{0}\left(1<x_{0}<\infty\right)$ such that

$$
C_{1}\left[1 /\left(x_{0}-x\right)^{1 / r-1}\right] \leqq f(x) \leqq C_{2}\left[1 /\left(x_{0}-x\right)^{1 / r-1}\right] \quad \text { as } x \uparrow x_{0},
$$

where $C_{1}$ and $C_{2}$ are positive constants.

If $u$ is an entire function of order 1 then $f$ is multivalued and

$$
C_{1} \ln \left[1 /\left(x_{0}-x\right)\right] \leqq f(x) \leqq C_{2} \ln \left[1 /\left(x_{0}-x\right)\right], \quad x \uparrow x_{0} .
$$

A simple test which excludes certain p.g.f.'s from class $\mathscr{I}$ (see Definition 1) requires merely checking the sign of the quantity

$$
e_{\pi}=\left[f^{\prime \prime}(\pi) / 2\right]^{2}-\left(f^{m}(\pi) / 3 !\right)\left(f^{\prime}(\pi) / 1\right)
$$

Thus we have

THEOREM 7. Let $f(x)$ be a p.g.f. and let $\pi$ denote the smallest positive root of $f(x)-x=0$. Assume that $f^{(\mathrm{iv})}(\pi)<\infty$. If $e_{\pi} \geqq 0$ then $f(x)$ is nonembeddable except if $f(x)$ is a linear fractional function.

The criterion of Theorem 7 is utilized most effectively by recourse to the following result. If $f(x)=\sum_{k=0}^{\infty} a_{k} x^{k}$ is a p.g.f. satisfying

$$
a_{k}^{2}-a_{k-1} a_{k+1} \geqq 0, \quad k=1,2,3, \ldots
$$

then $e_{\pi} \geqq 0$ and therefore $f$ is nonembeddable (see Theorem 8 of $\S 5$ ). A p.g.f. enjoying property (9) is said to be of class $\mathscr{R}$. Lemma 8 of $\S 5$ points out that $\mathscr{R}$ is closed with respect to pointwise multiplication. Putting together these facts we conclude that

$$
h(x)=C e^{\lambda(x-1)} \prod_{i=1}^{\infty}\left(q_{i}+p_{i} x\right)^{r_{i}} / \prod_{i=1}^{\infty}\left(1-\beta_{i} x\right)^{\alpha_{i}},
$$

where $C>0,0<p_{i}<1, q_{i}+p_{i}=1,0 \leqq \beta_{i}<1, \alpha_{i} \geqq 1, r_{i}$ are nonnegative integers, $i=1,2,3, \ldots, \lambda \geqq 0$ and $\sum_{i=1}^{\infty}\left(r_{i} p_{i}+\alpha_{i} \beta_{i}\right)<\infty$, is of class $\mathscr{R}$ and hence nonembeddable except if $h$ reduces to a linear fractional function.

Another criterion for nonembeddability is indicated in Corollary 3.

2. Some properties of the infinitesimal generating functions. We begin by reviewing some important facts from the theory of iteration of analytic functions as applied to the iterates of the p.g.f. $f(x)$. 
First consider the supercritical case $m=f^{\prime}(1)>1$. It is well known (see [3, p. 17] or [8]) that the limit

$$
A(x)=\lim _{n \rightarrow \infty} \frac{f_{n}(x)-\pi}{c^{n}}
$$

exists and the convergence is uniform in any disc $|x| \leqq 1-\varepsilon$. Evidently

$$
A(f(x))=c A(x), \quad A(\pi)=0, \quad A^{\prime}(\pi)=1
$$

and $A^{\prime}(x)>0$ for $0<x<1$. Thus the map $x \rightarrow w=A(x)$ maps the segment $[\pi, 1)$ monotonically on $[0, \infty)$ and the inverse map $w \rightarrow x=B(w)$ is analytic in a neighborhood of $[0, \infty)$, and there is a power series expansion $B(w)=\pi+w+b_{2} w^{2}+\cdots$ valid near $w=0$. If it is assumed that $f^{\prime \prime}(1)<\infty$ (actually the weaker condition $\sum a_{k} k \log k<\infty$ would be sufficient) then we can establish the existence of the limit

$$
A_{*}(x)=\lim _{n \rightarrow \infty} \frac{f_{-n}(x)-1}{m^{-n}}, \quad \pi<x \leqq 1,
$$

where $f_{-n}$ is the inverse function of $f_{n}$. The function $A_{*}(x)$ is continuous on $[\pi+\varepsilon, 1]$ and analytic at each point of $(\pi, 1)$, and satisfies

$$
A_{*}(f(x))=m A_{*}(x), \quad A_{*}(1)=0, \quad A_{*}^{\prime}(1)=1 .
$$

Also $w=A_{*}(x)$ provides a strictly monotone map of $(\pi, 1]$ onto $(-\infty, 0]$ and the inverse map $x=B_{*}(w)$ is analytic on $(-\infty, 0)$.

From (11) we have $A\left(f_{n}(x)\right)=c^{n} A(x), n=0,1,2, \ldots$, and hence the formula

$$
f_{t}(x)=B\left(e^{-\alpha t} A(x)\right), \quad t \geqq 0, \quad 0<x<1,
$$

provides an embedding (recall that $-\alpha=\log c$ ) of the iterates of $f(x)$ in a continuous one parameter semigroup of analytic functions which need not be p.g.f.'s. If there is any embedding of $f(x)$ in a continuous one parameter semigroup of p.g.f.'s then a simple argument based on (10) shows the embedding coincides with (14) for all rational $t$, hence by continuity for all $t$. The infinitesimal generator of the semigroup (14) is

$$
\left.\frac{\partial}{\partial t} f_{t}(x)\right|_{t=0}=u(x)=-\alpha \frac{A(x)}{A^{\prime}(x)}, \quad \pi<x \leqq 1 .
$$

In the subcritical case $m=f^{\prime}(1)<1$ we make the identifications $\pi=1, c=m$, and then provided $f^{\prime \prime}(1)<\infty$ (or again if only $\sum a_{k} k \log k<\infty$ ) it can be shown that the limit (10) exists uniformly in the full disc $|x| \leqq 1$, and satisfies (11). As before, if there is an embedding of $f(x)$ in a continuous one parameter semigroup of p.g.f.'s it must coincide with (14).

In view of (13) we deduce a second continuous embedding of $f_{n}(x), n=0,1,2, \ldots$, given by

$$
f_{t}^{*}(x)=B_{*}\left(e^{a t} A_{*}(x)\right), \quad 0<x<1 \quad(a=\log m),
$$

with corresponding infinitesimal generating function

$$
u_{*}(x)=a A_{*}(x) / A_{*}^{\prime}(x) .
$$


Of course, the representation (16) is available only in the supercritical case. It is important for our later purposes to relate $A(x)$ and $A_{*}(x)$. Before we can do this we need to elaborate various properties of $u(x)$. Inspection of (15) reveals that $u(x)>0$ for $0<x<\pi$ and $u(x)<0$ for $\pi<x<1$. Also, $u^{\prime}(\pi)=-\alpha$ and $u^{\prime}(1)=a$. Obvious manipulations on (11) and (15) yield the identity

$$
u(f(x))=f^{\prime}(x) u(x), \quad 0 \leqq x<1 .
$$

The analysis now proceeds separately for the supercritical and subcritical cases.

Case 1 (Subcritical).

LeMmA 1. If $f^{\prime}(1)=m<1$, then $A(x)$ (defined in (10)) and $A^{\prime}(x)$ are continuous on the closed segment $0 \leqq x \leqq 1$.

Proof. On the basis of the limit relation (10) and standard properties of convergence of sequences of analytic functions, we infer that

$$
f_{n}^{\prime}(x) / m^{n} \rightarrow A^{\prime}(x) \quad \text { uniformly for }|x|<1-\varepsilon .
$$

Note that $A\left(f_{n}(x)\right)=m^{n} A(x)$. Thus, if $x$ is restricted to any subinterval, say $\left[x^{*}, f\left(x^{*}\right)\right], x^{*}<1$ then $A\left(f_{n}(x)\right) \rightarrow 0=A(1)$ as $n \rightarrow \infty$ uniformly for $x \in\left[x^{*}, f\left(x^{*}\right)\right]$. But $f_{n}(x)$ converges uniformly to 1 for all $0 \leqq x \leqq 1$. Moreover, any sequence $\xi_{v} \rightarrow 1$, with $\xi_{1}>x^{*}$, can be expressed in the form $\xi_{v}=f_{n_{v}}\left(x_{v}\right)$ with $x_{v} \in\left[x^{*}, f\left(x^{*}\right)\right]$ and $n_{v} \rightarrow \infty$. Thereby we conclude that $A(x)$ is continuous at $x=1$.

Next observe that

$$
A^{\prime}\left(f_{n}(x)\right)=\left[m^{n} / f_{n}^{\prime}(x)\right] A^{\prime}(x) .
$$

A parallel argument based on this identity employing (19) shows that

$$
\lim _{x \rightarrow 1-} A^{\prime}(x)=A^{\prime}(1)=1 .
$$

Lemma 2. Suppose $f^{\prime \prime}(1)<\infty$, then $f_{n}^{\prime \prime}(x) / m^{n} \rightarrow A^{\prime \prime}(x)$ uniformly for $0 \leqq x \leqq 1$.

Proof. Twice differentiation of $f_{n}(x)=f\left(f_{n-1}(x)\right)$ and some rearrangements produce the formula

$$
\frac{f_{n}^{\prime \prime}(x)}{m^{n}}-\frac{f_{n-1}^{\prime \prime}(x)}{m^{n-1}}=f^{\prime \prime}\left(f_{n-1}(x)\right) \frac{\left[f_{n-1}^{\prime}(x)\right]^{2}}{m^{n}}+\frac{\left[f^{\prime}\left(f_{n-1}(x)\right)-m\right] f_{n-1}^{\prime \prime}(x)}{m^{n}} .
$$

Since $m \geqq f^{\prime}(x)$ on $[0,1]$ we see that

$$
\frac{f_{n}^{\prime \prime}(x)}{m^{n}}-\frac{f_{n-1}^{\prime \prime}(x)}{m^{n-1}} \leqq f^{\prime \prime}\left(f_{n-1}(x)\right) \frac{\left[f_{n-1}^{\prime}(x)\right]^{2}}{m^{n}} \leqq C m^{n} .
$$

Adding the inequalities of (22) for $n=1,2,3, \ldots$ yields the inequalities

$$
f_{n}^{\prime \prime}(x) / m^{n} \leqq C_{1}, \quad 0 \leqq x \leqq 1, \quad n=1,2, \ldots
$$

Now observe that

$$
\left|f^{\prime}\left(f_{n-1}(x)\right)-m\right| \leqq C_{2}\left|f_{n-1}(x)-1\right| \leqq C_{3} m^{n}, \quad 0 \leqq x \leqq 1 .
$$


With the aid of (22)-(24) we infer from (21) that $f_{n}^{\prime \prime}(x) / m^{n} \rightarrow A^{\prime \prime}(x)$ uniformly on $0 \leqq x \leqq 1$.

An analogous argument proves

LEMMA 3. If $f^{(\mathrm{iv})}(1)<\infty$ then $A^{(\mathrm{iv})}(x)$ is continuous on the closed unit segment $0 \leqq x \leqq 1$.

A simple consequence of Lemma 3 in view of the formula $u(x)=-\alpha\left(A(x) / A^{\prime}(x)\right)$ is the following

LEMma 4. If $f^{(\mathrm{iv})}(1)<\infty, f^{\prime}(1)=m<1$ then $u^{m}(x)$ exists and is continuous on the closed interval $x \in[0,1]$. Moreover, $u(x)$ and $-u^{\prime}(x)$ are positive for $0 \leqq x<1$ and $u(1)=0, u^{\prime}(1)=\log m$.

It is important to emphasize that the assertions of Lemma 4 prevail whether or not $f$ is embeddable.

Case 2 (Supercritical). The nature of $u(x)$ in the neighborhood of 1 in the supercritical case is intrinsically more complicated than for the subcritical case.

Define

$$
u(x)=-\alpha\left[A(x) / A^{\prime}(x)\right], \quad 0 \leqq x<1,
$$

where $A$ is constructed in (10). Without loss of generality (see (3) and its accompanying discussion), we may assume that $\pi=0$.

LEMma 5. Let $f$ be a p.g.f. such that $f^{\prime}(1)=m>1$ and $f^{\prime \prime}(1)<\infty$. Then $u(x)$ defined in (25) is continuous on $[0,1]$ vanishing at $\pi$ and 1 and $u^{\prime}(x)$ is uniformly bounded for $0 \leqq x<1$.

Proof. Since $A(x) \uparrow \infty$ as $x \uparrow 1-$ and the power series expansion of $A^{\prime}(x)$ displays only positive coefficients, a standard Abelian argument implies that $A(x) / A^{\prime}(x)$ is continuous on $[0,1]$ and vanishes at 1 . It remains to ascertain the nature of $u^{\prime}(x)$. We start with the identity

$$
u(f(x))=f^{\prime}(x) u(x)
$$

and write it in the form

$$
u(f(x))-u(x)=c(x) u(x)
$$

where $c(x)=f^{\prime}(x)-1$. Now iterate this relation and add to give

$$
u\left(f_{n}(x)\right)-u(x)=\sum_{k=0}^{n-1} c\left(f_{k}(x)\right) u\left(f_{k}(x)\right)
$$

Since $f_{n}(x) \rightarrow 0$, and $c(x)$ changes sign once as $x$ traverses $[0,1]$, we deduce that 
$\sum_{k=0}^{n} u\left(f_{k}(x)\right)$ is uniformly bounded for all $0 \leqq x \leqq 1$ and $n=1,2, \ldots$ Differentiate (26), and then iterate and sum to produce the formula

$$
u^{\prime}\left(f_{n}(x)\right)-u^{\prime}(x)=\sum_{k=0}^{n-1} d\left(f_{k}(x)\right) u\left(f_{k}(x)\right)
$$

where $d(x)=f^{\prime \prime}(x) / f^{\prime}(x)$. As $n \rightarrow \infty$, we obtain

$$
-\alpha-u^{\prime}(x)=\sum_{k=0}^{\infty} d\left(f_{k}(x)\right) u\left(f_{k}(x)\right)
$$

The right-hand expression is uniformly bounded over $0 \leqq x<1$ since $\sum_{k=0}^{\infty} u\left(f_{k}(x)\right)$ and $d(x)$ are bounded there. The proof of Lemma 5 is hereby complete.

Although $u^{\prime}(x)$ is continuous and uniformly bounded for $0 \leqq x<1$, in general, $\lim _{x \rightarrow 1-} u^{\prime}(x)$ does not exist. However, if $f$ is embeddable then $\lim _{x \rightarrow 1-} u^{\prime}(x)$ indeed exists in view of the fact that $u^{\prime}(x)$ admits a power series expansion with nonnegative coefficients (except for the constant term) convergent for $0 \leqq x<1$. We have thereby proved

COROLlaRY 1. Let the hypotheses of Lemma 5 hold. If $f$ is embeddable then $\lim _{x \rightarrow 1-} u^{\prime}(x)=\kappa$ exists and is finite.

The following lemma is useful.

Lemma 6. Assume that $\lim _{x \rightarrow 1-} u^{\prime}(x)=\kappa$ exists. If $f^{(r)}(1)<\infty$ for some integer $r(r \geqq 3)$ then $\lim _{x \rightarrow 1}-u^{(r-1)}(x)$ exists.

Proof. We prove the result in the case $r=3$, the general case is similar. Iterating the identity (cf. (27))

$$
u\left(f_{-1}(x)\right)-u(x)=e(x) u(x)
$$

yields

$$
\left(e(x)=f_{-1}^{\prime}(x)-1, f_{-1} \text { denotes the inverse function of } f\right)
$$

$$
u\left(f_{-n}(x)\right)-u(x)=\sum_{k=0}^{n-1} e\left(f_{-k}(x)\right) u\left(f_{-k}(x)\right), \quad 0 \leqq x<1 .
$$

We deduce paraphrasing the analysis of Lemma 5 and relying on the facts, that $f_{-n}(x)$ tends to 1 geometrically fast, $u(1)=0$ and $e(x)$ changes sign once, that $\sum_{k=0}^{\infty} u\left(f_{-k}(x)\right)$ converges uniformly on $[0,1]$. Analogous to (28) we obtain

$$
u^{\prime}\left(f_{-n-1}(x)\right)-u^{\prime}(x)=\sum_{k=0}^{n} u\left(f_{-k}(x)\right) \tilde{e}\left(f_{-k}(x)\right),
$$

where $\tilde{e}(x)=f_{-1}^{\prime \prime}(x) / f_{-1}^{\prime}(x)$. Letting $n \rightarrow \infty$ we have

$$
\kappa-u^{\prime}(x)=\sum_{k=0}^{\infty} u\left(f_{-k}(x)\right) \tilde{e}\left(f_{-k}(x)\right), \quad 0 \leqq x<1 .
$$

Differentiating this relation yields

(30) $-u^{\prime \prime}(x)=\sum_{k=0}^{\infty} u^{\prime}\left(f_{-k}(x)\right) \tilde{e}\left(f_{-k}(x)\right) f_{-k}^{\prime}(x)+\sum_{k=0}^{\infty} u\left(f_{-k}(x)\right) \tilde{e}^{\prime}\left(f_{-k}(x)\right) f_{-k}^{\prime}(x)$. 
Manifestly $f_{-k}^{\prime}(x) \rightarrow 1 / m^{k}$ as $x \rightarrow 1-$ for each $k$ and $f_{-k}^{\prime}(x) \leqq\left[f_{-1}^{\prime}\left(x_{0}\right)\right]^{k}$ for all $x_{0} \leqq x \leqq 1$ and any $x_{0}>0$. It follows by direct analysis of the terms in (30) that

$$
\lim _{x \rightarrow 1-}\left[-u^{\prime \prime}(x)\right]=\kappa \frac{m^{2}}{m-1} f_{-1}^{\prime \prime}(1)
$$

The proof of Lemma 6 is complete.

In the supercritical case provided $f^{\prime \prime}(1)<\infty$ we can construct

$$
A_{*}(x)=\lim _{n \rightarrow \infty} \frac{f_{-n}(x)-1}{m^{-n}}, \quad 0<x<1,
$$

with the properties $A_{*}(1)=0, A_{*}^{\prime}(1)=1, A_{*}(f(x))=m A_{*}(x)$ and such that $A_{*}$ is strictly monotone on $(0,1]$. Associated with $A_{*}(x)$ is the infinitesimal generating function

$$
u_{*}(x)=a A_{*}(x) / A_{*}^{\prime}(x)
$$

Paralleling the analysis of Lemmas $1-4$ we can demonstrate provided $f^{(\mathrm{iv})}(1)<\infty$ that $u_{*}^{\prime \prime}(s)$ exists and is continuous on the half open interval $(0,1]$. Since we will need the precise result we formulate it as a lemma.

Lemma 4'. Suppose $f^{\prime}(1)=m>1$. If $f^{(i)}(1)<\infty(i=2,3,4)$ then $A_{*}^{(i)}(x)$ and therefore $u_{*}^{(i-1)}(x)$ is continuous on the half open segment $(\pi, 1]$.

We sketch the proof. The proof of Lemmas 1 and 2 proceed mutatis mutandis in terms of $f_{-n}(x)$ for $f_{n}(x)$ and $A_{*}(x)$ for $A(x)$.

In the case of Lemma 2 the identity (21) is replaced by

$$
\frac{g_{n}^{\prime \prime}(x)}{m^{-n}}-\frac{g_{n-1}^{\prime \prime}(x)}{m^{-n+1}}=g^{\prime \prime}\left(g_{n-1}(x)\right) \frac{\left[g_{n-1}^{\prime}(x)\right]^{2}}{m^{-n}}+\frac{\left[g^{\prime}\left(g_{n-1}(x)\right)-m^{-1}\right] g_{n-1}^{\prime \prime}(x)}{m^{-n}}
$$

with $g_{n}=f_{-n}$.

We use the properties that $g=f_{-1}$ is monotone and concave on $[\pi+\varepsilon, 1]$ and that $g_{n}^{\prime}(x) / m^{-n}$ converges to $A_{*}^{\prime}(x)$ uniformly on $\pi+\varepsilon \leqq x \leqq 1$. It follows that $\left|g_{n}^{\prime \prime}(x)\right|$ $\leqq C_{1} m^{-n}$ on this interval. The rest of the proof proceeds with appropriate modifications as in the case of Lemma 3. The analogs of Lemmas 3 and 4 are proved by similar adaptations.

It is important to determine the relationship of the functions $A(x)$ and $A_{*}(x)$. It is easier to do this for the corresponding inverse functions $B(w)$ and $B_{*}(w)$.

THEOREM 1. Let $f$ be a p.g.f. satisfying $f^{\prime \prime \prime}(1)<\infty, f^{\prime}(1)=m>1, f(0)=0$ and $f^{\prime}(0)=c>0$. Let $B(w)$ and $B_{*}(w)$ denote the inverse functions of $A(x)$ and $A_{*}(x)$ respectively associated with $f$. 
(i) There exists a bounded continuous function $L(\lambda)$ defined on the half plane $\operatorname{Re} \lambda \geqq 0, \lambda \neq 0$ satisfying

$$
L(m \lambda) \equiv L(\lambda)
$$

Moreover

$$
B_{*}(-\lambda)=B\left[L(\lambda) / \lambda^{\gamma}\right], \quad \operatorname{Re} \lambda \geqq 0,
$$

where

$$
\gamma=\frac{\log (1 / c)}{\log m}=\frac{\alpha}{a}=\frac{-u^{\prime}(0)}{u^{\prime}(1)}
$$

(ii) If $\lim _{x \rightarrow 1}-u^{\prime}(x)$ exists then $L(\lambda)$ is identically constant and thus

$$
A(x)=\kappa /\left[-A_{*}(x)\right]^{\gamma}, \quad \pi<x<1 .
$$

(iii) If $f$ is embeddable then (34) holds.

Proof. The classical martingale convergence theorem for supercritical branching processes implies, in particular, that

$$
\lim _{n \rightarrow \infty} f_{n}\left(e^{-\lambda / m^{n}}\right)=\phi(\lambda) \quad \text { uniformly for } \operatorname{Re} \lambda \geqq 0
$$

(see [3, p. 15]).

The solution of

$$
\frac{A^{\prime}(x)}{A(x)}=\frac{-\alpha}{u(x)}, \quad 0<x<1, \quad A(0)=0, \quad A^{\prime}(0)=1
$$

admits the expression

$$
A(x)=\frac{\kappa}{(1-x)^{\gamma}} \exp \left(\int_{1}^{x}\left[\frac{-\alpha}{u(\xi)}+\frac{\gamma}{\xi-1}\right] d \xi\right), \quad|x|<1 .
$$

In view of the representation $f_{n}(x)=B\left(e^{-\alpha n} A(x)\right)$ (cf. (14)) and the convergence relation (35) we have

$$
\phi(\lambda)=\lim _{n \rightarrow \infty} B\left(\frac{\kappa}{\left(1-e^{-\lambda / m^{n}}\right)^{\gamma} e^{\alpha n}} \exp \left[\int_{1}^{e^{-\lambda / m^{n}}}\left[\frac{-\alpha}{u(\xi)}+\frac{\gamma}{\xi-1}\right] d \xi\right]\right) .
$$

But $\left(1-e^{-\lambda / m^{n}}\right)^{\gamma} e^{\alpha n} \rightarrow \lambda^{\gamma}$ and consequently

$$
\lim _{n \rightarrow \infty} \kappa \exp \left(\int_{1}^{e^{-\lambda / m^{n}}}\left[\frac{-\alpha}{u(\xi)}+\frac{\gamma}{\xi-1}\right] d \xi\right)=L(\lambda)
$$

exists and obeys the stated properties as substantiated by straightforward considerations. Thus

$$
\phi(\lambda)=B\left[L(\lambda) / \lambda^{r}\right]
$$


A similar argument based on the representations $f_{n}(x)=B_{*}\left(e^{a n} A_{*}(x)\right)$ (cf. (16)) and the formula

leads to the result

$$
A_{*}(x)=-(1-x) \exp \left(\int_{1}^{x}\left[\frac{a}{u_{*}(\xi)}-\frac{1}{\xi-1}\right] d \xi\right)
$$

$$
\phi(\lambda)=\lim _{n \rightarrow \infty} f_{n}\left(e^{-\lambda / m^{n}}\right)=\lim _{n \rightarrow \infty} B_{*}\left(e^{a n} A_{*}\left(e^{-\lambda / m^{n}}\right)=B_{*}(-\lambda) .\right.
$$

(The smoothness properties of $u_{*}(\xi)$ at 1 enunciated in Lemma $4^{\prime}$ need to be used in order to justify (39).) Comparing (38) and (39) validates (33). The proof of part (i) is complete.

(ii) Imposing the hypothesis of the existence of $\lim _{x \rightarrow 1-} u^{\prime}(x)$ we see according to Lemma 6 that $\lim _{x \rightarrow 1}-u^{\prime \prime}(x)$ is defined. Then examination of (37) reveals that $L(\lambda) \equiv \kappa=$ a positive constant.

(iii) For the case when $f$ is embeddable we proved in Corollary 1 that $u^{\prime}(1-)$ exists and consequently also $u^{\prime \prime}(1-)$ exists (Lemma 6). Invoking the conclusion of (ii) yields the desired result.

The next theorem asserts that if $f$ is a generating function of class $\mathscr{S}$ (see the definition following Theorem $\mathrm{A}, \S 1$ ), then $L(\lambda)$ is not constant. In this case the relation (32) compels that $L(\lambda)$, although bounded, is highly oscillatory as $\lambda \rightarrow 0$.

THEOREM 2. Let $f(x)$ be a p.g.f. satisfying the conditions of Theorem 1 of class $\mathscr{S}$ (see Definition 2) and not a linear fractional function, then $L(\lambda)$ determined in (37) is not a constant. In particular, for $u$ defined by $(25), \lim _{x \rightarrow 1-} u^{\prime}(x)$ does not exist.

Proof. For $f$ of class $\mathscr{S}$ or more generally for $f$ merely analytic at 1 then $B_{*}(w)$ is analytic in a neighborhood about $w=0$. If $L(\lambda) \equiv \kappa$ then

$$
B_{*}(-\lambda)=B\left(\kappa / \lambda^{\gamma}\right)
$$

obtains and the right-hand function can be used to produce an analytic continuation of $B_{*}(\lambda)$ to certain regions of the complex plane. (Recall that $B(w)$ is always analytic in a neighborhood of $[0, \infty)$.) It is also known that $B_{*}(\lambda)$ satisfies the functional equation

$$
f\left(B_{*}(w)\right)=B_{*}(m w), \quad|w|<\rho \text { for some } \rho>0 .
$$

Examination of the proof of Theorem A given in [8] reveals the validity of the following statement. Let $f, B$ and $B_{*}$ enjoy the properties: (a) $f \in \mathscr{S}$, (b) $B(w)$ and $B_{*}(w)$ are analytic at $w=0$, (c) $B$ and $B_{*}$ are connected by the relation (40) and (d) $f$ and $B_{*}$ obey the functional equation (41). Then $f$ is a linear fractional function.

This assertion implies the first statement of the theorem. The final statement is a consequence of Theorem 1, part (ii).

Theorem 2 and other evidence suggests the conjecture that in the supercritical case $f$ is embeddable iff $L(\lambda) \equiv$ constant. The validity of the identity $B_{*}(-\lambda)$ $=B\left(\kappa / \lambda^{\gamma}\right)$ bears interesting probabilistic consequences pertaining to the limiting 
density of the family of the random variables $X_{n} m^{-n}$. It is not difficult to prove that $L(\lambda) \equiv$ constant iff $u^{\prime}(1-)$ exists. The implication that $u^{\prime}(1-)$ exists entails $L(\lambda) \equiv$ constant is subsumed in the statement of Theorem 1, part (i). Conversely suppose $L(\lambda) \equiv c$, then the identity (34) holds. It then follows that $u_{*}(x)=u(x)$ for $0<x<1$. But $u_{*}^{\prime}(1)$ certainly exists (see Lemma 1$)$ and therefore $u^{\prime}(1-)$ exists.

Observe that

$$
\left[L(\lambda) / \lambda^{\gamma}\right]=A\left(B_{*}(-\lambda)\right), \quad 0<\lambda<\infty,
$$

is completely monotone. This conclusion ensues from the fact that $A$ admits a power series expansion with nonnegative coefficients and $B_{*}(-\lambda)=\phi(\lambda)$ is manifestly completely monotone.

3. Characterization of the linear fractional function within the class of embeddable p.g.f. Consider the supercritical branching process, i.e., $f^{\prime}(1)=m>1$. Let $\pi$, as before, denote the smallest positive root of the equation $f(x)-x=0$. We have $\pi<1$ and without restricting generality (see (3) and its accompanying discussion), we may take $\pi=0$. Also assume $f^{\prime}(0)=c>0$.

The parameters $\alpha=\log \left(1 / f^{\prime}(0)\right)$ and $a=\log f^{\prime}(1)$ and their ratio $\gamma=\alpha / a$ play an important role in the analysis concerning the supercritical branching process. When $f$ is embeddable and $u(x)$ represents the associated infinitesimal generating function (see (15)) then, of course $u^{\prime}(0)=-\alpha$ and $u^{\prime}(1)=a$.

THEOREM 3. Let $f$ be a p.g.f. such that $f^{\prime \prime}(1)<\infty$. If $f$ is embeddable in a continuous time branching process with infinitesimal generating function $u(x)$, then $0<\gamma \leqq 1$ and equality $(\gamma=1)$ holds iff $u(x)=-\alpha x(1-x)$ or equivalently $f$ is the linear fractional function $f(x)=\left(e^{-\alpha} x\right) /\left[1-\left(1-e^{-\alpha}\right) x\right]$.

Proof. The expansion of $u(x)$ has the form

$$
u(x)=\lambda\left(\sum_{i=1}^{\infty} p_{i} x^{i}-x\right)
$$

where $\lambda>0, p_{i} \geqq 0$ and $\sum_{i=1}^{\infty} p_{i}=1$. Observe that $u^{\prime}(1)=\lambda \sum_{i=2}^{\infty}(i-1) p_{i} \geqq \lambda\left(1-p_{1}\right)$ while $u^{\prime}(0)=\lambda\left(p_{1}-1\right)$. Thus, $0<\gamma \leqq 1$ and equality manifestly occurs iff $p_{i}=0$ for $i=3,4, \ldots$.

The same method proves

THEOREM 4. Let $u(x)$ be the infinitesimal generating function of a continuous time Markov branching process and let

$$
u(x)=\lambda(h(x)-x)=\lambda\left(p_{0}+\left(p_{1}-1\right) x+p_{2} x^{2}+p_{3} x^{3}+\cdots\right)
$$

where $p_{i} \geqq 0, \sum_{i=0}^{\infty} p_{i}=1$ with $h^{\prime}(1)<\infty$. If $p_{0}=p_{2}=p_{3}=\cdots=p_{k}=0$ and $p_{k+1}>0$ then $0<\gamma \leqq 1 / k$ and $\gamma=1 / k$ iff $u(x)=-\alpha x\left(1-x^{k}\right)$.

The generating function $f(x)$ corresponding to $u(x)=-\alpha x\left(1-x^{k}\right)$ has the form $g(x ; k)=\left[(1-p)^{1 / k} x\right] /\left(1-p x^{k}\right)^{1 / k}, 0<p<1$ or $g(x ; k)=\phi^{-1}\left(g(\phi(x ; 1))\right.$ where $\phi(x)=x^{k}$ 
and $g(x ; 1)=[(1-p) x] /(1-p x)$. We will see later $(\S 4)$ that the special p.g.f. semigroups $g_{t}(x ; k)$ occupy a special position among those where $f_{t}(x)$ is analytic at 1 .

It is of some interest to note that the parameter $\gamma=\alpha / a$ for the p.g.f. $f(x)=$ $(1-p)^{1 / 2} /(1-p x)^{1 / 2}$ also ranges from 0 to 1 as $p$ varies from $1 / 3$ to 1 , although this p.g.f. is nonembeddable.

4. Proof of Theorem 6 and supplements. Throughout this section $f(x)$ will represent a p.g.f. regular at 1 . Let $x_{0}>1$ be the smallest positive singular point of $f(x)$. Pringsheim's theorem about power series with nonnegative coefficients guarantees the existence of a singularity $x_{0}$ located on the positive axis, conceivably at $\infty$. The same theorem informs us that the radius of convergence of $f(x)=\sum_{k=0}^{\infty} a_{k} x^{k}$ equals $x_{0}$.

Suppose $f(x)$ is embeddable and let $u(x)$ denote the corresponding infinitesimal generating function. Because of Theorem $\mathrm{A}$ we know that $f$ is not an entire function and therefore $1<x_{0}<\infty$. Appealing to Theorem 1, part (ii) and relations (34), (15) and (13) we conclude that $u(x)$ is analytic in a circle of radius exceeding 1. Again invoking Pringsheim's theorem and noting that the coefficients of $u(x)$ $=\sum_{r=0}^{\infty} u_{r} x^{r}$ (except for $u_{1}$ ) are nonnegative, we find that $u(x)$ is analytic in a circle about the origin whose radius extends to the first singular point of $u(x)$ on the positive axis. We are now prepared to prove the following lemma of independent interest.

LEMMA 7. Let $f$ be an embeddable p.g.f. regular at 1 . Let $x_{0}\left(x_{0}>1\right)$ be the smallest positive singular point of $f(x)$ and suppose $f(x) \uparrow \infty$ as $x \uparrow x_{0}$. Then $u(x)$ is an entire function and $f(x) \leqq C /\left(x_{0}-x\right), 0 \leqq x<x_{0}$ for some constant $C$.

Proof. Consider first the case where $f^{\prime}(1)=m>1$. Consulting (18) and passing to inverse functions gives

$$
u(g(y))=g^{\prime}(y) u(y), \quad g(y)=f_{-1}(y) .
$$

Note because $f$ strictly increases to $\infty$ as $x \uparrow x_{0}$ that $g$ is analytic on $[1, \infty)$ and $g^{\prime}(y)>0$ on this segment. Since $g(y)<y$ for $y>1$ the functional relation (42) serves to provide an analytic continuation of $u$ to the entire positive axis. Application of the Pringsheim theorem to $u(x)$ implies that $u$ is entire.

Next observe that

$$
u(f(x))=\sum_{i=0}^{\infty} u_{i}[f(x)]^{i}=f^{\prime}(x) u(x), \quad 0<x<x_{0}
$$

and $u$ being nontrivial by stipulation (since $f(x) \not \equiv x$, see the start of $\S 1$ ), we obtain some $r \geqq 2$ for which $u_{r}>0$.

Since $f(x) \uparrow \infty$ as $x \uparrow x_{0}, 0<u\left(x_{0}\right)<\infty$ (actually $u(x)>0$ for all $x>1$ ), and $u_{i} \geqq 0$ for all $i \geqq 2$ we deduce on the basis of (43) the inequality

$$
d \leqq f^{\prime}(x) /[f(x)]^{r}, \quad x_{0}-\varepsilon \leqq x<x_{0},
$$


provided $\varepsilon$ is specified sufficiently small, where $d$ is an appropriate positive constant. Integrating (44) from $x$ to $x_{0}$ and simplifying yields

(45) $f(x) \leqq \frac{C}{\left(x_{0}-x\right)^{1 /(r-1)}} \leqq \frac{C}{\left(x_{0}-x\right)}, \quad x_{0}-\varepsilon \leqq x<x_{0}, \quad(C=$ a constant $)$.

The proof of Lemma 7 is complete in the supercritical case.

The subcritical case $(m<1)$ can be reduced to the supercritical case by exploiting the device of (2). Note here that $f(x) \uparrow \infty$ as $x \uparrow \infty$ and therefore the existence of a second fixed point is assured.

The critical case $(m=1)$ is handled by employing the same methods. In this case the infinitesimal generator is defined by the formula $u(x)=1 / A^{\prime}(x)$ where now

$$
A(x)=\lim _{n \rightarrow \infty}\left\{\frac{1}{1-f_{n}(x)}-\frac{1}{1-f_{n}(0)}\right\}
$$

(see [6] or [7]) satisfies the functional equation

$$
A(f(x))=A(x)+A(f(0)) .
$$

When $f$ is embeddable we find again that $u$ is analytic at $1 ; u(f(x))=f^{\prime}(x) u(x)$ and the arguments proceed as before. The proof of Lemma 6 is now complete.

Proof of Theorem 6. (Consult $\S 1$ for the precise statement of the theorem.) The hypotheses of Lemma 7 are certainly fulfilled and therefore $u(x)$ is entire. Since $f^{\prime}(x)$ grows like $c_{0} /\left(x_{0}-x\right)^{\delta+1}$ as $x \uparrow x_{0}$ and

$$
\sum_{i=0}^{\infty} u_{i}[f(x)]^{i}=u(f(x))=f^{\prime}(x) u(x), \quad 0<x<x_{0},
$$

holds where $u_{i} \geqq 0$ for $i \geqq 2$ and $0<u\left(x_{0}\right)<\infty$ it follows from examination of the growth behavior of $u(f(x))$ for $x \uparrow x_{0}$ that $u$ is a polynomial of precise degree $r \geqq 2$ where $r \delta=\delta+1$ or equivalently

$$
\delta=1 /(r-1)
$$

A further differentiation of (46) yields

$$
u^{\prime}(f(x))=u^{\prime}(x)+\left[f^{\prime \prime}(x) / f^{\prime}(x)\right] u(x) .
$$

The right-hand side is manifestly single valued in the neighborhood of $x_{0}$ because of the assumptions on $f(x)$. Now consider

$$
u^{\prime}(f(x))=u_{1}+2 u_{2} f(x)+\cdots+(r-1) u_{r-1}[f(x)]^{r-2}+r u_{r}[f(x)]^{r-1} .
$$

Because of (47) we see that $[f(x)]^{r-1}$ is also single valued at $x_{0}$. Therefore

$$
F(x)=2 u_{2} f(x)+\cdots+u_{r-1}(r-1)[f(x)]^{r-2}
$$

must be single valued at $x_{0}$. But near $x_{0}$ the term involving $[f(x)]^{r-2}$ dominates and its expansion about $x_{0}$ behaves like

$$
(r-1) u_{r-1} c_{0} /\left(x_{0}-x\right)^{(r-2) / r-1}
$$


This behavior is impossible unless $u_{r-1}=0$ (recall that $c_{0} \neq 0$ ). Repeating this reasoning with the remaining terms of (49) we deduce recursively that $u_{r-2}=0, \ldots$, $u_{2}=0$. Thus $u(s)$ has the form

$$
u(s)=u_{1} s+u_{r} s^{r}
$$

which is the infinitesimal generating function of the p.g.f. semigroup (6). The proof of Theorem 6 is complete.

Proposition 1 of $\S 1$ is the same as Lemma 7 apart from the assertions connected with (7) and (8). These are proved adapting appropriately the reasoning leading to (45). We omit the details.

The analysis of Theorem 6 can be considerably refined. For example the hypothesis can be somewhat relaxed concerning the nature of the singularity of $x_{0}$ with the conclusion unchanged. We do not attempt to exhaust all its ramifications. Review of the preceding analysis also reveals the following facts:

(i) Let $u$ be an infinitesimal generating function of a supercritical continuous time branching process. Suppose $u$ is regular at 1 but not entire. Then $f_{t}(x)$ for any fixed $t$ is regular at 1 but does not tend to $\infty$ as $x$ increases to its first singularity on the positive axis.

(ii) A version of Theorem 6 exists replacing the condition $f(0)=0$ by $f(\pi)=\pi$ for some $0<\pi \leqq 1$ where $\pi$ has the usual interpretation.

5. Some further criteria for nonembeddability. The criteria of Theorems 7 and 8 below can frequently be easily checked thereby eliminating certain p.g.f.'s as members of the class $\mathscr{I}$.

THEOREM 7. Let $f(x)$ be a p.g.f. and let $\pi$ denote the smallest positive root of $f(x)-x=0$. Assume that $f^{(\mathrm{iv})}(\pi)<\infty$ and $f^{\prime}(\pi)>0$. If

$$
e_{\pi}=\left[\frac{f^{\prime \prime}(\pi)}{2}\right]^{2}-\frac{f^{m}(\pi)}{3 !} \cdot \frac{f^{\prime}(\pi)}{1} \geqq 0,
$$

then $f(x)$ is nonembeddable unless $f(x)$ is a linear fractional function.

REMARK. If $f^{\prime}(1)=m>1$ then $\pi<1$ and the assumption $f^{(\text {iv })}(\pi)<\infty$ is automatically fulfilled. In the case $m \leqq 1$ then $\pi=1$ and $f^{(\text {iv })}(1)<\infty$ indeed imposes a restriction on $f$.

Proof. Case (i) $m \neq 1$. Let $A(x)$ be determined according to (10). Thus $A^{(\mathrm{iv})}(\pi)$ exists (if $\pi=1$, use Lemma 4) and $A(s)$ satisfies

$$
A(f(x))=c A(x), \quad A(\pi)=0, \quad A^{\prime}(\pi)=1, \quad c=f^{\prime}(\pi) .
$$

Successive differentiations in (51) and evaluations at $\pi$ finally produce the formulas

$$
A^{\prime \prime}(\pi)=\frac{f^{\prime \prime}(q)}{c(1-c)}, \quad A^{\prime \prime}(\pi)=\frac{3\left[f^{\prime \prime}(\pi)\right]^{2}+(1-c) f^{\prime \prime}(\pi)}{c(1+c)(1-c)^{2}}
$$

Substituting and simplifying yields the identity

$$
\left[\frac{A^{\prime \prime}(\pi)}{2}\right]^{2}-\frac{A^{\prime \prime \prime}(\pi)}{3 !} \frac{A^{\prime}(\pi)}{1}=\frac{1}{c^{2}\left(1-c^{2}\right)}\left[\left(\frac{f^{\prime \prime}(\pi)}{2}\right)^{2}-\frac{f^{\prime \prime}(\pi)}{3 !} \frac{f^{\prime}(\pi)}{1}\right]
$$


If $f$ is embeddable then the infinitesimal generating function can be expressed in the form

$$
u(x)=-\alpha\left[A(x) / A^{\prime}(x)\right], \quad(\alpha=\log (1 / c)) .
$$

Since $u(x)$ generates a semigroup of p.g.f.'s we must have $0<u^{\prime \prime}(\pi)$ unless $f$ is a linear fractional p.g.f. (see Lemma 4). An easy calculation taking account of the normalizations $A(\pi)=0$ and $A^{\prime}(\pi)=1$ gives

$$
u^{\prime \prime \prime}(\pi)=12 \log \frac{1}{c}\left[A^{\prime}(\pi) \frac{A^{\prime \prime \prime}(\pi)}{3 !}-\left[\frac{A^{\prime \prime}(\pi)}{2}\right]^{2}\right] .
$$

Comparing (52) and (54) and recalling the fact $0<c<1$ we see that condition (50) entails the inequality $u^{\prime \prime \prime}(\pi) \leqq 0$. It follows that $f$ is nonembeddable except if $f$ is a linear fractional function.

Case (ii). $m=f^{\prime}(1)=1$. Suppose to the contrary that $f(x)$ is embeddable in a semigroup $f_{t}(x)$ of p.g.f.'s with infinitesimal generating function $u(x)$. Then it is easily checked (see [3, p. 107]) that

$$
A(x)=\int_{0}^{x} \frac{1}{u(\xi)} d \xi, \quad 0<x<1,
$$

satisfies $A\left(f_{t}(x)\right)=A(x)+t$ and, in particular

$$
A(f(x))=A(x)+1 .
$$

Observe from the properties of $u(x)$ that $A^{\prime}(x)>0$ and $A^{\prime \prime}(x)>0$ for $0<x<1$ and $A(x)$ is analytic in the unit circle. Subject to the hypothesis of $f^{(\mathrm{iv})}(1)<\infty$ we established in [7] that apart from an additive constant $A(x)$ admits the representation

$$
A(x)=1 /(1-x)+\left(e_{1} / a\right) \log (1-x)+\psi(x)+d,
$$

where $e_{1}$ is defined in (50), $a=f^{\prime \prime}(1) / 2, d$ is a constant and $\psi^{\prime}(x)$ is bounded on $0 \leqq x<1$. Differentating (57) and consulting (55) we obtain

$$
1 / u(x)=1 /(1-x)^{2}-e_{1} / a(1-x)+\psi^{\prime}(x), \quad 0<x<1 .
$$

Since $\psi^{\prime}(x)$ is uniformly bounded for $0 \leqq x<1$, the relation (58) clearly implies that $u^{\prime \prime \prime}(1-) / 3 !=-e_{1} / 3 a$ which is nonpositive by virtue of the hypothesis. But, excluding the linear fractional case, $f$ embeddable requires that $u^{\prime \prime \prime}(1-)>0$. Thus we have a contradiction and the proof of the theorem is complete.

The following theorem presents a useful sufficient condition which guarantees the validity of (50).

THEOREM 8. Let $f(s)=\sum_{k=0}^{\infty} a_{k} s^{k}$ be a p.g.f. such that

$$
a_{k}^{2}-a_{k-1} a_{k+1} \geqq 0, \quad k=1,2,3, \ldots
$$

Then

$$
\left[\frac{f^{(r)}(x)}{r !}\right]^{2}-\frac{f^{(r-1)}(x)}{(r-1) !} \frac{f^{(r+1)}(x)}{(r+1) !} \geqq 0 \quad \text { for all } 0 \leqq x<1 \quad \text { and } \quad r=1,2, \ldots
$$


Definition 3. A p.g.f. satisfying (59) is said to be of class $\mathscr{R}$.

Proof. Consider

$$
f(y+x)=\sum_{k=0}^{\infty} \frac{f^{(k)}(x)}{k !} y^{k}=\sum_{k=0}^{\infty} b_{k} y^{k} \quad \text { for } 0<x<1,
$$

where the expansion is of course, valid at least for $|y|<1-x$. A straightforward computation gives

$$
b_{n}=\sum_{k=0}^{\infty} a_{k} x^{k-n}\left(\begin{array}{l}
k \\
n
\end{array}\right), \quad n=0,1,2, \ldots
$$

Executing some obvious rearrangements we obtain

$$
b_{n+m+1}=\sum_{k=0}^{\infty} a_{k} x^{k-n-m-1} \sum_{l=0}^{k}\left(\begin{array}{l}
l \\
n
\end{array}\right)\left(\begin{array}{c}
k-l \\
m
\end{array}\right)=x^{-n-m-1} \sum_{k=0}^{\infty}\left(\begin{array}{l}
k \\
n
\end{array}\right) \sum_{l=0}^{\infty} a_{l+k}\left(\begin{array}{l}
l \\
m
\end{array}\right) x^{k} x^{l} .
$$

We define the kernels $R(k, l)=a_{l+k} x^{k} x^{l}$ and $U(l, m)=\left(\begin{array}{l}l \\ m\end{array}\right)$ for all nonnegative integers $k, l$ and $m$. Set

$$
V(k, m)=\sum_{l=0}^{\infty} R(k, l) U(l, m) .
$$

The hypothesis guarantees the determinant inequality

$$
\left|\begin{array}{ll}
R\left(k_{1}, l_{1}\right) & R\left(k_{1}, l_{2}\right) \\
R\left(k_{2}, l_{1}\right) & R\left(k_{2}, l_{2}\right)
\end{array}\right| \geqq 0 \quad \text { for all } 0 \leqq k_{1}<k_{2} \quad \text { and } \quad 0 \leqq l_{1}<l_{2} .
$$

A simple argument also gives the inequality

$$
\left|\begin{array}{ll}
U\left(l_{1}, m_{1}\right) & U\left(l_{1}, m_{2}\right) \\
U\left(l_{2}, m_{1}\right) & U\left(l_{2}, m_{2}\right)
\end{array}\right| \geqq 0 \quad \text { for all } 0 \leqq l_{1} \leqq l_{2} \quad \text { and } \quad 0 \leqq m_{1} \leqq m_{2} .
$$

An application of the Cauchy Binet identity for determinants of products of matrices applied to $(60)$ yields the relation

$$
\left|\begin{array}{ll}
V\left(k_{1}, m_{1}\right) & V\left(k_{1}, m_{2}\right) \\
V\left(k_{2}, m_{1}\right) & V\left(k_{2}, m_{2}\right)
\end{array}\right|=\sum_{l_{1}<l_{2}}\left|\begin{array}{ll}
R\left(k_{1}, l_{1}\right) & R\left(k_{1}, l_{2}\right) \\
R\left(k_{2}, l_{1}\right) & R\left(k_{2}, l_{2}\right)
\end{array}\right|\left|\begin{array}{ll}
U\left(l_{1}, m_{1}\right) & U\left(l_{1}, m_{2}\right) \\
U\left(l_{2}, m_{1}\right) & U\left(l_{2}, m_{2}\right)
\end{array}\right|
$$

In view of (61) and (62) we infer that the left hand expression in (63) is nonnegative provided $0 \leqq k_{1}<k_{2}$ and $0 \leqq m_{1}<m_{2}$. Clearly we may express $b_{n+m}$ in the form

$$
b_{n+m+1}=y^{-n-m-1} \sum_{k=0}^{\infty} V(k, m) U(k, n) .
$$

The same procedure using the nonnegative of the determinants (63) just established and (62) shows that

$$
\left|\begin{array}{ll}
b_{n_{1}+m_{1}+1} & b_{n_{1}+m_{2}+1} \\
b_{n_{2}+m_{1}+1} & b_{n_{2}+m_{2}+1}
\end{array}\right| \geqq 0, \quad 0 \leqq n_{1}<n_{2},
$$

and in particular $b_{n+m+1}^{2} \geqq b_{n+m} b_{n+m+2}$. The proof is complete.

Applying a similar and actually easier argument, we can prove 
Lemma 8. If $f$ and $g$ are of class $\mathscr{R}$ then $f \cdot g$ is also of class $\mathscr{R}$ (see [5, Chapter 8]).

EXAMPLE. It can be checked directly that

$$
\begin{array}{ll}
f(s)=e^{\lambda(s-1)}, & \lambda \geqq 0, \\
f(s)=\frac{(1-\beta)^{\alpha}}{(1-\beta s)^{\alpha}}, & (\alpha>1,0<\beta<1)
\end{array}
$$

and

$$
f(s)=(q+p s)^{r}, \quad 0<p<1, \quad p+q=1,
$$

$\boldsymbol{r}$ a positive integer are all of class $\mathscr{R}$. Application of Lemma 8 and obvious limiting procedures shows that the p.g.f.

$$
h(s)=C e^{\lambda(s-1)} \prod_{i=1}^{\infty}\left(q_{i}+p_{i} s\right)^{r_{i}} / \prod_{i=1}^{\infty}\left(1-\beta_{i} s\right)^{\alpha_{i}} \quad \text { is of class } \mathscr{R},
$$

provided $0 \leqq p_{i}<1, q_{i}+p_{i}=1,0 \leqq \beta_{i}<1, \alpha_{i} \geqq 1, i=1,2,3, \ldots, \lambda \geqq 0$ and

$$
\sum_{i=1}^{\infty}\left(r_{i} p_{i}+\alpha_{i} \beta_{i}\right)<\infty
$$

Theorems 7 and 8 tell us that a p.g.f. of the form (66) is embeddable only in the special circumstance that $h(s)$ reduces to a linear fractional function.

Part of the argument of Theorem 7 also proves the following corollary.

CoROLlary 2. Let $f$ be a p.g.f. satisfying $f^{(\mathrm{iv})}(1)<\infty$. If $f$ is embeddable in a semigroup of p.g.f.'s and $f^{\prime}(1)=1$ and

$$
e_{1}=\left[f^{\prime \prime}(1) / 2\right]^{2}-f^{m}(1) / 3 !=0
$$

then $f$ is a linear fractional function.

6. Univalence and embeddability. We examine only the supercritical case subject to the normalization $f(0)=0, f^{\prime}(0)=c>0$. We will show that a necessary condition that $f$ be embeddable is that $f$ define a univalent mapping of the open unit circle into itself (Corollary 3 below). The associated function $A(x)$ (see (10)) is also endowed with special mapping properties as attested to by the following theorem.

THEOREM 9. Let the normalizations of (1) prevail with $\pi=0$. If $u(x)$ defined in (15) generates a semigroup of p.g.f.'s then $A(x)$ determines a univalent star shaped mapping of $|x|<1$.

Proof. We pointed out earlier that the infinitesimal generating function of the positive semigroup has the expression

$$
u(x)=\lambda(h(x)-x)
$$

where $\lambda>0$ and $h$ is a p.g.f. Because of the normalization $f(0)=0$ we also have 
$h(0)=0$. It is clear from $(10)$ that $A(x)$ is analytic throughout $|x|<1$. Notice by comparing (67) and (15) that

$$
x\left[A^{\prime}(x) / A(x)\right]=d /(1-g(x))
$$

where $g(x)=h(x) / x$ and $d=\alpha / \lambda>0$. Since $g$ is a p.g.f. obviously $|g(x)|<1$ for $|x|<1$. Therefore, on $|x|<1$

$$
\operatorname{Re}\left\{x\left[A^{\prime}(x) / A(x)\right]\right\}=d\left(\operatorname{Re}[1-g(\bar{x})] /|1-g(x)|^{2}\right) \geqq 0
$$

or equivalently

$$
\frac{\partial}{\partial \theta} \arg A(x) \geqq 0, \quad \text { for } x=\rho e^{i \theta}, \quad 0<\rho<1,
$$

which means that $A(x)$ winds in the same direction as $x$ traverses clockwise the circle of radius $\rho$. (This property signifies that $A(x)$ determines a star shaped map of the open unit disc.) Moreover a simple application of Róuche's theorem yields

$$
\frac{1}{i} \int_{|z|=\rho} \frac{A^{\prime}(z)}{A(z)} d z=d \frac{1}{i} \int_{|z|=\rho} \frac{d z}{z-h(z)}=2 \pi .
$$

The preceding facts easily imply that $A(z)$ is univalent in $|z|<1$.

COROLlary 3. If $f$ is a p.g.f. satisfying (1) with $\pi=0$ and embeddable then $f$ is univalent in the unit circle.

This inference is a simple consequence of Theorem 9 and the functional relationship (11).

Remark. Consider the situation where the smallest positive fixed point $\pi$ does not occur at zero i.e., $f(\pi)=\pi, 0 \leqq \pi<1$. In this case the conclusion of Theorem 9 is modified so that if $f$ is embeddable then $A(x)$ is univalent in the circle $|x-\pi|$ $<1-\pi$. The proof requires simply noting the relationship of $f$ and $\hat{f}$ and the associated semigroups $f_{t}(x)$ and $\hat{f}_{t}(x)$ described in (3).

The analog of Corollary 3 asserts that when $f$ is embeddable then $f$ is univalent in the circle $|x-\pi|<1-\pi$.

There are many other smoothness characteristics for $\hat{f}$ inherent to the property that $f$ is embeddable. Theorem 9 and Corollary 3 are typical. Some additional smoothness characteristics of $f$ of class $\mathscr{I}$ are discussed by Kurtz [10].

\section{REFERENCES}

1. I. N. Baker, Fractional iteration near a fixed point of multiplier 1, J. Austral. Math. Soc. 4 (1964), 143-148.

2. T. E. Harris, Some mathematical models for branching processes, Proc. 2nd Berkeley Sympos., Univ. of California Press, Berkeley, California, 1951.

3. - The theory of branching processes, Springer, New York, 1963.

4. E. Jabotinsky, Analytic iteration, Trans. Amer. Math. Soc. 108 (1963), 457-477.

5. S. Karlin, Total positivity and applications, Stanford Univ. Press, Stanford, California, 1967. 
6. S. Karlin and J. McGregor, Spectral representation of branching processes. II, Z. Wahrscheinlichkeitstheorie und Verw. Gebiete. 5 (1966), 34-54.

7. - Properties of the stationary measure of the critical case simple branching process, Ann. Math. Statist. 38 (1967), 977-991.

8. - Embedding iterates of analytic functions with two fixed points into continuous groups, Trans. Amer. Math. Soc. 132 (1968), 137-145.

9. G. Koenigs, Recherches sur les intégrales de certaines équations fonctionnelles, Ann. Sci. École Norm. Sup. 1, Supplement (1884), 2-41.

10. T Kurtz, Some topics in branching processes, Ph.D. Dissertation, Stanford Univ., Stanford, Calif., 1967.

11. G. Szekeres, Regular iteration of real and complex functions, Acta Math. 100 (1960), 203-258.

12. E. C. Titchmarsh, The theory of functions, Oxford Univ. Press, New York, 1939.

STANFORD UNIVERSITY,

StANFord, CALIFornia 